\title{
Detecting Extrasolar Planet Transits from the South Pole
}

\author{
Douglas A. Caldwell \\ SETI Institute, 2035 Landings Dr. Mountain View, CA, 94043 USA \\ William J. Borucki ${ }^{1}$, Robert L. Showen ${ }^{2}$,Jon M. Jenkins ${ }^{3}$, Laurance \\ Doyle $^{3}$, Zoran Ninkov ${ }^{4}$, Michael Ashley ${ }^{5}$ \\ ${ }^{1}$ NASA Ames Research Center, ${ }^{2}$ Raytheon, ${ }^{3}$ SETI Institute, ${ }^{4}$ Rochester \\ Institute of Technology, ${ }^{5}$ University of New South Wales
}

\begin{abstract}
We have developed and tested a wide-field photometer to detect extrasolar planet transits from the South Pole. The discovery of transiting planets for which masses can be measured by radial velocity is vital to constrain the models of planet formation and evolution. Short of going to space, the South Pole is the best site from which to carry out a such a survey. Based on results from the Doppler velocity surveys and the Vulcan transit search, we expect to detect 10 to 15 transiting planets in two years of operation at the South Pole.
\end{abstract}

\section{Extrasolar Planet Transits}

The recent deluge of extrasolar planet discoveries by Doppler velocity surveys has resulted in a wealth of valuable science (see http://www.obspm.fr/planets for the latest catalog). Planets have been found with masses ranging from 0.1 to 10 Jupiter masses, with a wide range of eccentricities, and with orbital semimajor axes ranging from $a=0.05$ to 5 times the Earth-Sun distance $(1 \mathrm{AU})$. Several planetary systems have been discovered, with the hint that many of the other known planets are also in multiple-planet systems (Fischer et al. 2001). One particularly surprising class of planets is the set of close-in giant planets found in 3 to 5 day period orbits around approximately $1 \%$ of the stars surveyed (Mayor \& Queloz 1995; Butler et al. 2001). Because of their small orbital radii, there is a significant chance $\left(R_{*} / a \sim 10 \%\right)$ that the plane of the orbit will be aligned such that the planet passes in front of, or "transits," its star as seen from Earth. During transit, the planet blocks a fraction of the star's light equal to the the ratio of the areas of the planet and $\operatorname{star}\left(R_{p}^{2} / R_{*}^{2} \sim 1 \%\right.$ for a Jupiter-Sun transit), causing a measurable dimming of the star. The duration and shape of the transit signal depend on the orbital parameters of the planet and the brightness distribution across the face of the star.

Only one of the known extrasolar planets transits its star: HD 209458b. Photometric observations of its transits have resulted in the first measurements of an extrasolar planet's size and density (Charbonneau et al. 2000; Henry et al. 2000), limits on the size of any moons or rings that may be present (Brown 
et al. 2001), and the first detection of a planetary atmosphere outside of our Solar System (Charbonneau et al. 2002). The results coming from the study of HD 209458b have fueled the race to detect more transiting exoplanets. There are several current ground-based searches for transits and several satellite missions scheduled for launch: COROT, Kepler, and Eddington.

All transit searches need to account for the rarity of observable transits. The number of transits expected for a given search can be expressed as:

$$
N_{\text {det }}=1\left(\frac{N_{*}}{2700}\right)\left(\frac{P_{d}}{0.5}\right)\left(\frac{P_{p}}{0.0075}\right)\left(\frac{P_{a}}{0.1}\right) P_{w i n} P_{\sigma}
$$

where $N_{*}$ is the number of stars monitored, $P_{d}$ is the probability that a given target star is a dwarf (i.e., a main-sequence star, small enough for a transit to be visible), $P_{p}$ is the probability that a given star has a planet, $P_{a}$ is the probability of sufficient alignment for transits to be visible from Earth, $P_{\text {win }}$ is the probability that a set of transits from a given planet will occur during the observations, and $P_{\sigma}$ is the noise-dependent detection probability of a set of transits. Clearly, $N_{*}, P_{\text {win }}$, and $P_{\sigma}$ are the quantities most under the control of the observer. $N_{*}$ and $P_{\sigma}$ are determined by the design of the photometer and the choice of star-fields.

In order to reliably detect a transiting planet, multiple transits must be seen. A set of three transits is the minimum needed to measure, and then confirm, the planet's orbital period. The window function, $P_{w i n}$, is the fraction of phases at a given orbital period for which at least three transits occur in the set of observations. $P_{w i n}$ depends strongly on the fraction of each day the stars are observed and the total length of the campaign. Space missions can achieve $P_{\text {win }}=1$ by observing continuously for more than three times the longest period planet to be detected. On Earth, the closest one can come to continuous coverage comes through taking advantage of the long polar night.

\section{South Pole Transit Search}

The unique conditions and location of the South Pole make it the best place on Earth to detect transiting short period extrasolar planets. First, the long winter night allows the randomly-phased transits to be most efficiently be detected. Given perfect weather, a search could reach $P_{\text {win }}=1$ for planets with periods up to 1 month during the 3 month night. At low or mid-latitude sites observations are missed for a large portion of each day while the Sun is up. Combined with observations missed due to weather, this means that only a small fraction of the total time available is actually used. For example, the Vulcan project (Borucki et al. 2001) observed a field in Cygnus over 83 days during the summer of 1999 . The weather permitted observations on 56 nights, covering only $15 \%$ of the total time, or $60 \%$ of the dark time. In addition, the detection of a set of transits depends on the distribution in time of the observations: Vulcan is relatively insensitive to planets with periods near 1 day. During winter at the South Pole there is no 24-hour day-night cycle; only weather limits the observations. The night time weather at the South Pole is suitable for observing about $50 \%$ of the time (Barnaby et al. 2001). Figure 1 presents the three-transit window function, $P_{w i n}$, for three data sets: 28 days of modeled South Pole observations with $50 \%$ 


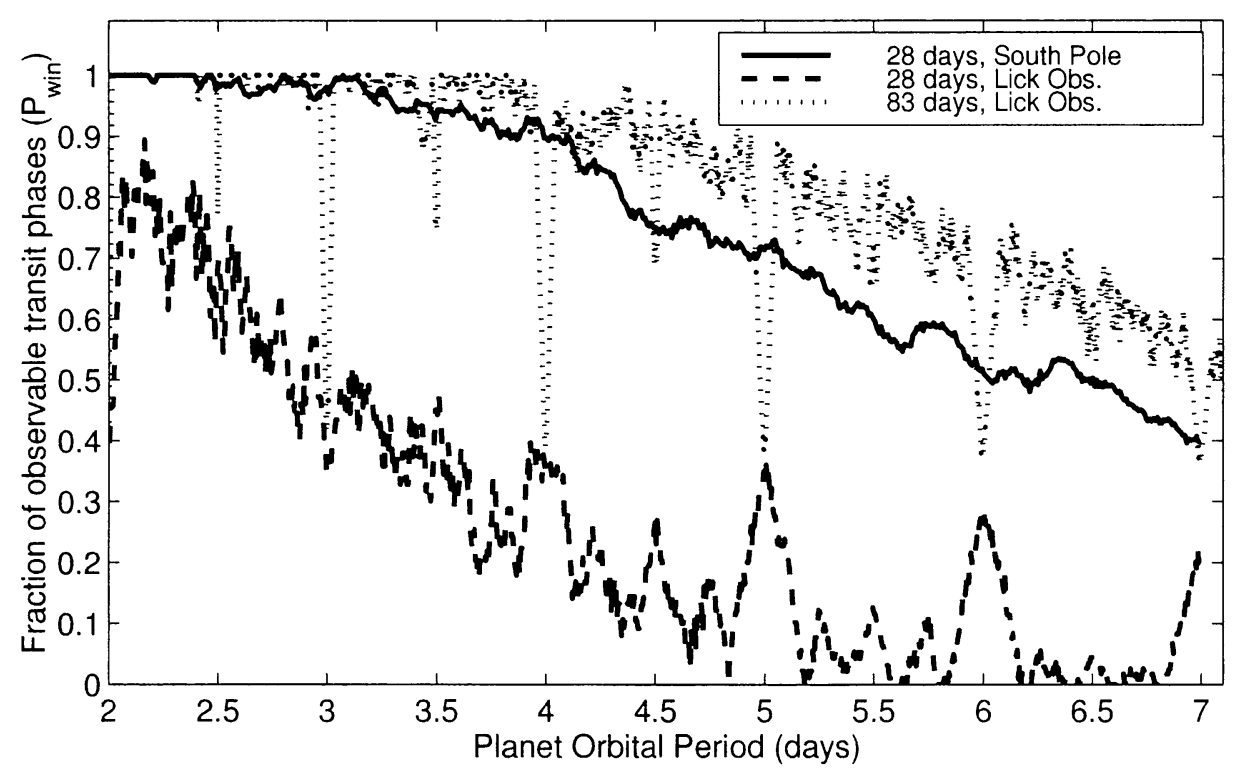

Figure 1. $\quad P_{w i n}$ versus period for one month of model South Pole data and one and three months of actual observations made by the Vulcan project at Lick Observatory, a mid-latitude site in the USA. The phase coverage from one month at the South Pole is comparable to three months from a mid-latitude site.

coverage, 83 days of actual Vulcan observations, and the most well covered 28 day subset of the Vulcan observations. For planets with periods between 2.5 and 7 days, one month of observations at the South Pole gives a comparable 3 -transit detection rate to three months of mid-latitude observations.

A second major advantage of the South Pole is that the constant altitude of a star field eliminates the large daily flux variation due to atmospheric extinction changes as the stars rise and set. These changes are generally on the order of $10 \%$, much larger than a transit signal. Extinction modeling removes most of this effect, but requires careful determination of color-dependent terms for thousands of stars. The major complication of the airmass induced brightness change for transit detection is that it occurs on the same time scale as a transit (several hours). Therefore, any errors in the correction add noise at the time scale of the signal, the worst situation for signal detection. At the South Pole, the only changes in extinction are due to changes in the atmospheric conditions, which are smaller and on varying time scales.

The South Pole offers several other practical advantages as an observing site for an extrasolar planet transit search. It is a dark site at an altitude of $2830 \mathrm{~m}$. There is no rain, very little snow fall, and very little wind. There are many well-situated rich star fields, as the Galactic plane crosses the sky with a peak elevation near $65^{\circ}$ in the vicinity of the Southern Cross. These star fields are readily observable from Australia from February through June, allowing 
us ample time to analyze our data and prepare candidate lists for follow-up observations.

\section{South Pole Deployment}

In February 2001 we deployed a prototype system to the South Pole. The photometer, one used earlier for Vulcan, consisted of a $4 \mathrm{k} \times 4 \mathrm{k}$ CCD camera, stopped-down optics appropriate for daylight testing, a fully steerable mount, a heating and insulation system, and a control and data acquisition computer. The test deployment was quite successful. The CCD camera and optics performed without troubles. The heating/insulation system worked well at $\sim 33 \%$ power with the outside temperatures around $-40^{\circ} \mathrm{C}$. Perhaps most importantly, we found that the technical support and logistics are available to successfully operate a small telescope at the South Pole

We are currently constructing a transit search system to be deployed overwinter at the South Pole. The photometer is based on the Vulcan system, using the same CCD camera that was deployed in our prototype and a fast $\mathrm{f} / 1.5$ $300 \mathrm{~mm}$ focal length lens, to give a wide field-of-view $\left(7^{\circ} \times 7^{\circ}\right)$. The photometer will be mounted on the G-mount, a telescope mount designed and built by the Mt. Stromlo Observatory specifically for use in the extreme cold of the South Pole winter (Storey et al. 2000). The G-mount has been operating successfully at the South Pole for several seasons. After over-winter tests in 2003, we expect to deploy a fully operating system in the austral summer of 2003-4. The system will operate for two winter seasons. It will monitor $\sim 10,000$ stars from $V=9$ to 13 magnitude continuously for 6 weeks with the goal to monitor two fields per dark season. We expect to detect 10 to 15 transiting extrasolar planets in two years of observations. In addition, we will generate a well-sampled photometric database for tens of thousands of Southern Hemisphere stars, including the discovery of $\sim 200$ eclipsing binaries (based on results from Vulcan), and a record of winter observing conditions at the South Pole.

\section{References}

Barnaby, D., et al. 2001, PASP, submitted

Borucki, W. J., et al. 2001, PASP, 113, 439

Brown, T. M., Charbonneau, D., Gilliland, R. L., Noyes, R. W., \& Burrows, A. 2001, ApJ, 552, 699

Butler, R. P., et al. 2000, in Planetary Systems in the Universe: Observations, Formation and Evolution, in ASP Conference Series, ed. A. Penny, P. Artymowicz, A.-M. Lagrange, \& S. Russell, 1

Charbonneau, D., Brown, T., Noyes, R., \& Gilliland, R. 2002, ApJ, 568, 377

Charbonneau, D., Brown, T., Latham, D., \& Mayor, M. 2000, ApJ, 529, L45

Fischer, D., et al. 2001, ApJ, 551, 1107

Henry, G. W., Marcy, G. W., Butler, R. P., \& Vogt, S. S. 2000, ApJ, 529, L41

Mayor, M., \& Queloz, D. 1995, Nature, 378, 355

Storey, J. W., Ashley, M. C., \& Burton, M. G. 2000, Proc. SPIE, 4008, 1376 